GLASNIK MATEMATIČKI

Vol. 39(59)(2004), $27-30$

\title{
ON GENERALIZED DERIVATIONS AS HOMOMORPHISMS AND ANTI-HOMOMORPHISMS
}

\author{
NADEEM-UR-REHMAN \\ Birla Institute of Technology and Science, India
}

\begin{abstract}
The concept of derivations as well as generalized derivations (i.e. $I_{a, b}(x)=a x+x b$, for all $a, b \in R$ ) have been generalized as an additive function $F: R \longrightarrow R$ satisfying $F(x y)=F(x) y+x d(y)$ for all $x, y \in R$, where $d$ is a nonzero derivation on $R$. Such a function $F$ is said to be a generalized derivation. In the present paper it is shown that: if $R$ is 2 -torsion free prime ring, $I \neq 0$ an ideal of $R$ and $F$ a generalized derivation of $R$ such that either $F(x y)=F(x) F(y)$ or $F(x y)=F(y) F(x)$ for all $x, y \in I$, then $R$ is commutative.
\end{abstract}

\section{INTRODUCTION}

Throughout the present paper $R$ will denote an associative ring with center $Z(R)$. For any $x, y \in R$, the symbol $[x, y]$ stands for the commutator $x y-y x$. Recall that a ring $R$ is called prime if for any $a, b \in R, a R b=(0)$ implies that either $a=0$ or $b=0$. An additive mapping $d: R \longrightarrow R$ is called a derivation if $d(x y)=d(x) y+x d(y)$ holds for all $x, y \in R$. For a fixed $a \in R$ the mapping $I_{a}: R \longrightarrow R$ given by $I_{a}(x)=[x, a]$ is a derivation which is said to be inner derivation.

An additive function $F_{a, b}: R \longrightarrow R$ is called generalized inner derivation if $F_{a, b}(x)=a x+x b$ for some fixed $a, b \in R$. It is straight forward to note that if $F_{a, b}$ is a generalized inner derivation, then for any $x, y \in R$, $F_{a, b}(x y)=F_{a, b}(x) y+x I_{b}(y)$ where $I_{b}$ is an inner derivation. In view of the above observation, the concept of generalized derivation is introduced as follows: an additive mapping $F_{a, b}: R \longrightarrow R$ is called a generalized derivation associated with a derivation $d$ if $F(x y)=F(x) y+x d(y)$ for all $x, y \in R$.

2000 Mathematics Subject Classification. 16W25, 16N60, 16 U80.

Key words and phrases. Prime rings, generalized derivations, torsion free rings, homomorphisms and anti-homomorphisms. 
Generally we do not mention the derivation $d$ associated with a generalized derivation $F$ rather prefer to call simply a generalized derivation. One may observe that the concept of generalized derivation includes the concept of derivation and generalized inner derivations, also of the left multipliers when $d=0$. Hence it should be interesting to extend some results concerning to these notations to generalized derivations. Recently some authors have also studied generalized derivation in the theory of operator algebras and $C^{*}-$ algebras ( see for example [5]).

Let $S$ be a nonempty subset of $R$ and $F$ be a generalized derivation of $R$. If $F(x y)=F(x) F(y)$ or $F(x y)=F(y) F(x)$ for all $x, y \in S$, then $F$ is called a generalized derivation which acts as homomorphisms or anti-homomorphisms, respectively.

In [2] Bell and Kappe proved that if a derivation $d$ of a prime ring $R$ which acts as homomorphisms or anti-homomorphisms on a nonzero right ideal of $R$ then $d=0$ on $R$. Further Ashraf et al [1] obtained this result for $(\sigma, \tau)$-derivation. In the present paper our objective is to extend this result for generalized derivation acting on ideals in prime ring.

Throughout the present paper we shall make extensive use of the following basic commutator identities without any specific mention:

$$
[x y, z]=x[y, z]+[x, z] y, \text { and }[x, y z]=y[x, z]+[x, y] z
$$

The proof of the following lemma can be found in [6].

LEMMA 1.1. If a prime ring $R$ contains a nonzero commutative right ideal, then $R$ is commutative.

THEOREM 1.2. Let $R$ be a 2-torsion free prime ring and $I$ be a nonzero ideal of $R$. Suppose $F: R \longrightarrow R$ is a nonzero generalized derivation with $d$

(i) If $F$ acts as a homomorphism on $I$ and if $d \neq 0$, then $R$ is commutative.

(ii) If $F$ acts as an anti-homomorphism on $I$ and if $d \neq 0$, then $R$ is commutative.

ProOF. ( $i$ ) If $F$ acts as a homomorphism on $I$, then we have

$$
F(x y)=F(x) y+x d(y)=F(x) F(y) \text { for all } x, y \in I .
$$

For any $x, y, z \in I$, we find that

$$
F(x y z)=F(x y) z+x y d(z) \text { for all } x, y, z \in I .
$$

On the other hand

$$
F(x y z)=F(x) F(y z)=F(x) F(y) z+F(x) y d(z) \text { for all } x, y, z \in I .
$$

On comparing (2) and (3), we get $(F(x)-x) y d(z)=0$ for all $x, y, z \in I$, and hence $(F(x)-x) I d(z)=(0)$ for all $x, z \in I$. Thus, primeness of $R$ forces that either $(F(x)-x)=0$ or $d(z)=0$. If $d(z)=0$ for all $z \in I$, then $d=0$, a contradiction. On the other hand if $F(x)=x$ for all $x \in I$, then 
$x y=F(x y)=F(x) y+x d(y)$ for all $x, y \in I$ and hence we find that $x d(y)=0$ i.e. $I d(y)=0$. Again since $I \neq 0$, and $R$ is prime we get $d(y)=0$ for all $y \in I$ and hence $d=0$, again a contradiction.

(ii) If $F$ acts as an anti-homomorphism

$$
F(x y)=F(x) y+x d(y)=F(y) F(x) \text { for all } x, y \in I .
$$

Replacing $x$ by $x y$ in (4) and using (4), we get

$$
x y d(y)=F(y) x d(y) \text {, for all } x, y \in I .
$$

Now, replace $x$ by $z x$ in (5), to get

$$
z x y d(y)=F(y) z x d(y) \text {, for all } x, y, z \in I .
$$

Left multiplying (5) by $z$, we obtain

$$
z x y d(y)=z F(y) x d(y) \text {, for all } x, y, z \in I \text {. }
$$

Comparing (6) and (7), we find that $[F(y), z] x d(y)=0$, for all $x, y, z \in I$ i.e. $[F(y), z] I d(y)=(0)$. Thus for each $y \in I$, by primeness of $R$ either $[F(y), z]=0$ or $d(y)=0$. Now, let $A=\{y \in I \mid[F(y), z]=0$, for all $z \in I\}$, $B=\{y \in I \mid d(y)=0\}$. Thus $A$ and $B$ are additive subgroups of $I$ and $I=A \cup B$. But a group can not be a union of two proper subgroups and hence $I=A$ or $I=B$. If $I=B$ then $d(y)=0$ for all $y \in I$ and hence $d=0$, a contradiction. On the other hand, if $I=A$, then $[F(y), z]=0$, for all $y, z \in I$. Now, replace $y$ by $y z$ to get $[y, z] d(z)+y[d(z), z]=0$. Again replacing $y$ by $x y$ we get $[x, z] y d(z)=0$ for all $x, y, z \in I$ i.e. $[x, z] \operatorname{Id}(z)=(0)$. Thus primeness $R$ implies that for each $z \in I$ either $[x, z]=0$ or $d(z)=0$. Hence again applying Brauer's trick, we find that either $d(z)=0$ for all $z \in I$ or $[x, z]=0$, for all $x, z \in I$. If $d(z)=0$ for all $z \in I$ then $d=0$. Now, if $[x, z]=0$ for all $x, z \in I$ then by Lemma 1.1 we get the required result. This completes the proof of the theorem.

\section{ACKNowledgements.}

The author is greatly indebted to the referee for several useful suggestions and valuable comments which led to improvement the exposition.

\section{REFERENCES}

[1] M. Ashraf, N. Rehman and M.A. Quadri, On $(\sigma, \tau)$-derivations in certain classes of rings, Rad. Math. 9 (1999), 187-192.

[2] H.E. Bell and L.C. Kappe, Rings in which derivations satisfy certain algebric conditions, Acta Math. Hung. 53 (1989), 339-346.

[3] H.E. Bell and W.S. Martindale, Centralizing mappings of semiprime rings, Canad. Math. Bull. 30 (1987), 91-101.

[4] I.N. Herstein, Topics in Ring theory, Univ. Chicago press, Chicago 1969.

[5] B. Havala, Generalized derivations in rings, Comm. Algebra 26 (1998), 1147-1166.

[6] J.H. Mayne, Centralizing mappings of prime rings, Canad. Math. Bull. 27 (1984), $122-126$. 
[7] N. Rehman, On commutativity of rings with generalized derivations, Math. J. Okayama Univ. 44 (2002), 43-49.

[8] M.S. Yenigul and N. Argac, On prime and semi prime rings with $\alpha$-derivations, Turkish J. Math 18 (1994), 280-284.

Department of Mathematics

Birla Institute of Technology and Science

Pilani 333031

Rajasthan, India

Received: 27.09 .2002

Revised: 15.01 .2003 